\title{
A rare complication of Laparoscopic Sleeve Gastrectomy: Gastro- Esophageal Transection
}

\author{
Kenneth D' Cruz ${ }^{1}$, Digant Pathak ${ }^{2}$, Thimmaraj Nayaka N S 3 \\ ${ }^{I}$ (Dept. Of Surgical Gastroenterology, Narayana Hrudayalaya, Bangalore, India) \\ ${ }^{2}$ (Dept. Of Surgical Gastroenterology, Narayana Hrudayalaya, Bangalore, India) ${ }^{3}$ (Dept. Of Surgical \\ Gastroenterology, Narayana Hrudayalaya, Bangalore, India)
}

\begin{abstract}
Laparoscopic sleeve gastrectomy is a commonly done restrictive bariatric surgical procedure. It is considered to be a relatively safe and technically simple bariatric operation. This report describes the case of a 40 year old morbidly obese female who had complete gastro-esophageal transection during laparoscopic sleeve gastrectomy. She underwent laparotomy with resection of the gastro-esophageal junction and Roux-en-Y esophago-jejunostomy as a single staged corrective procedure. We are reporting the case for its extreme rarity. To our knowledge it is the first case report of such a complication which was managed as a single stage corrective procedure.
\end{abstract}

Keywords: Sleeve gastrectomy, gastro-esophageal transection, complications of sleeve gastrectomy, Bariatric Surgery

\section{Introduction}

Laparoscopic sleeve gastrectomy, first described as a modification of the biliopancreatic diversion-duodenal switch is now a popular stand-alone operation for the treatment of morbid obesity. It has an acceptable morbidity and long-term weight loss is comparable with the laparoscopic Rouxen-Y gastric bypass and adjustable gastric band [1].

Although rare, leakage and bleeding from the staple line are the commonly reported complications. Esophageal transection during sleeve gastrectomy has been reported recently for the first time, which was managed in multiple stages over a long duration [2]. We are reporting similar complication, with transection at the Gastro-esophageal junction. Corrective surgery was performed in a single stage.

\section{Case Report}

Our patient is 40 year old female with BMI of 48 , who underwent laparoscopic sleeve gastrectomy. During surgery, the gastric calibration tube could not be negotiated into the stomach. In the post-operative period, she developed persistent non-bilious vomiting and retching. On the fifth postoperative day a gastrograffin swallow study was done under fluoroscopy, which showed a dilated esophagus and holding up of contrast at the gastro-esophageal junction, without entry of contrast distally (Fig.1). At flexible endoscopy the scope could not be negotiated beyond the oesophagus, which was dilated and full of saliva. Plain CT scan of chest and abdomen showed a dilated esophagus with no air in the gastric tube, suggestive of complete blockage at the gastro-esophageal junction. There was also evidence of aspiration pneumonitis in the right lung (Fig.2).

She was then transferred to our institution by the primary surgeon. At admission, her pulse rate was normal and she was afebrile. Her pulmonary status was optimised and she was subjected to an upper midline laparotomy on post-operative day 8. At exploration, two staple lines were found. The distal staple line was across the antrum of the stomach, $4 \mathrm{cms}$ proximal to the pylorus, extending to the lesser curve. The proximal staple line extended from the lesser curve of the stomach across the gastro-esophageal junction to the first short gastric vessels (Fig.3). The left gastric artery and hepatic artery were intact. The distal staple line was resected leaving $3 \mathrm{cms}$ of antrum proximal to the pylorus. The remnant of gastro-esophageal junction attached to the first short gastric vessels was mobilized. The oesophagus was transected immediately proximal to the gastroesophageal junction with an endoscopic linear cutter $(60 \mathrm{~mm}$, blue stapler). A jejunal Roux-en- Y loop was prepared and brought up through a window in the mesocolon. The Orville (Covedien, Ireland) was passed via the mouth and brought out through the oesophageal staple line and the anvil secured with a purse string suture. The oesophago-jejunal anastomosis was performed with the EEA 25 stapler (Covedien, Ireland), and the jejunum closed with the endoscopic linear cutter. A $120 \mathrm{cms}$ long trans-anastomotic nasojejunal feeding tube of $12 \mathrm{~F}$ was inserted. A 16F closed suction drain was placed near the esohago-jejunal anastomosis, and brought out through the left flank. 
Postoperatively, she received partial parenteral nutritional support with added enteral tube feeding. On the $10^{\text {th }}$ postoperative day, a gastrograffin swallow was done, which showed prompt passage of dye into the jejunum with no evidence of leak. The nasojejunal feeding tube was removed and she commenced oral intake of liquids. She was discharged on the $11^{\text {th }}$ postoperative day.

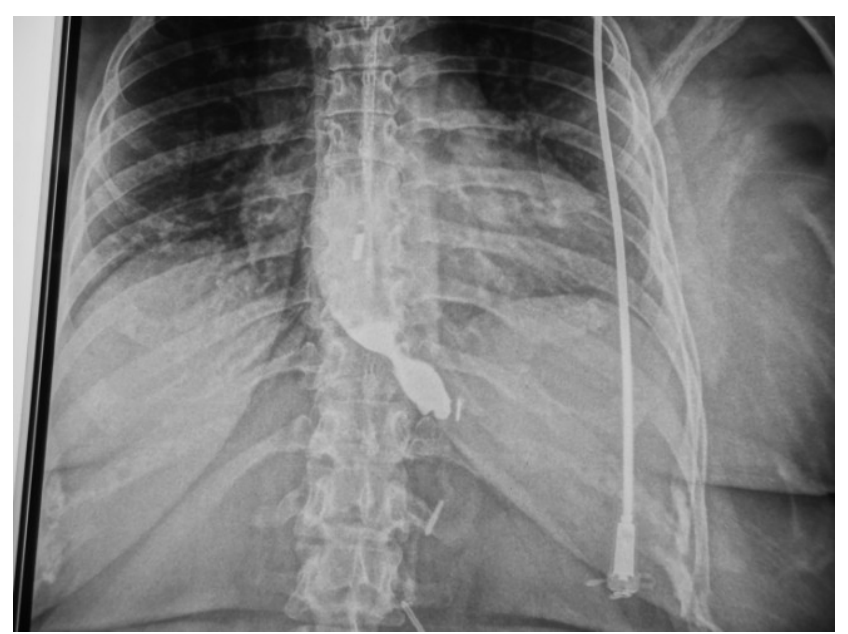

Fig. 1- Gastrograffin swallow showing a dilated esophagus and holding up of contrast at the gastro-esophageal junction, without entry of contrast distally.

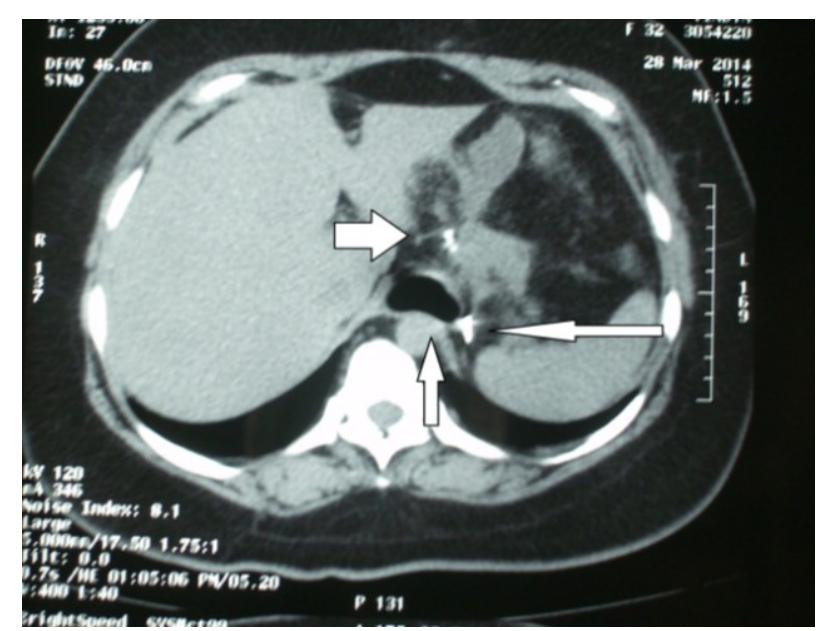

Fig. 2- NCCT scan of abdomen and thorax showing dilated esophagus (short arrow), gasless abdomen and staple line along the gastric tube (bold arrow) and along the lower end of esophagus (long arrow)

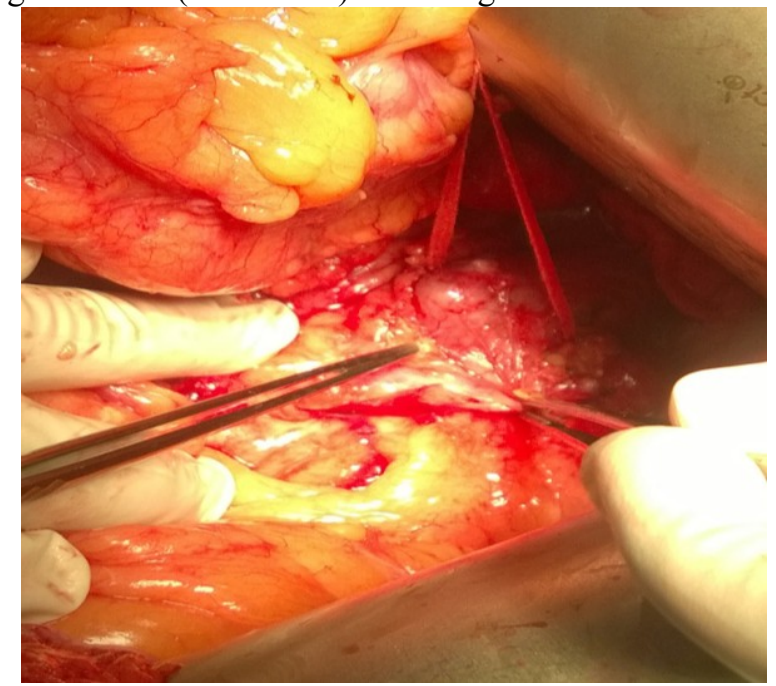

Fig.3- Intraoperative photograph showing blind end esophagus along with staple line and bougie inside held with umbilical tape 


\section{Discussion}

Laparoscopic sleeve gastrectomy is an established surgical approach for the treatment of obesity. The procedure has seen a surge in popularity because of its perceived technical simplicity, feasibility and good outcomes [3].

Laparoscopic sleeve gastrectomy is claimed to have a favorable complication profile even in high risk patients [4]. Staple line bleeding and leaks are among the complications that have been reported as being of the highest immediate concern to the surgeon. Esophageal transection as a complication of LSG has been reported only once in the English literature [2].This is the first case report of transection at the gastro-esophageal junction during laparoscopic sleeve gastrectomy. As there was no leak or sepsis, a single stage corrective procedure was feasible.

Proper training is a minimum prerequisite for a bariatric surgeon. A gastric sleeve should always be performed with an adequate sized gastric calibration tube in place to avoid excessive narrowing of the stomach tube: as well as esophageal or gastric transection. If this tube cannot be placed, an endoscope should be inserted and the sleeve gastrectomy carried out.

We support the view of Weiner et al that as Laparoscopic sleeve gastrectomy is being adopted by more bariatric surgeons; there is definite need for the procedure to become standardized to achieve similar results across different practices [5].

\section{Conclusion}

As in all surgical procedures, if certain principles are not followed, even a simple procedure can end up with major complications, as in this case of gastro-esophageal transection during laparoscopic sleeve gastrectomy. We advocate proper training as a minimum prerequisite for a novice bariatric surgeon and gastric sleeve should always be formed with adequate sized gastric calibration tube in place to avoid esophageal or gastric transection during the surgery. In addition, practice of performing leak test during the surgery will also be an invaluable tool to avoid such complication.

\section{References}

[1] Bohdjalian A, Langer FB, Shakeri-Leidenmuhler S, et al. Sleeve gastrectomy as sole and definitive bariatric procedure: 5-year results for weight loss and ghrelin. Obes Surg. 2010; 20:535-540

[2] Meshikhes and Al-Saif. Iatrogenic oesophageal transection during laparoscopic sleeve gastrectomy. BMJ Case Reports 2014; doi: $10.1136 /$ bcr-2013-201260

[3] Raul J. Rosenthal. International Sleeve Gastrectomy Expert Panel Consensus Statement: best practice guidelines based on experience of $>12,000$ cases. Surgery for Obesity and Related Diseases.2012; 8: 8-19.

[4] Shi X, Karmali S, Sharma A, et al. A review of laparoscopic sleeve gastrectomy for morbid obesity. Obes Surg. 2010; 20:11711177.

[5] Weiner RA, Weiner S, Pomhoff I, et al. Laparoscopic sleeve gastrectomy-influence of sleeve size and resected gastric volume. Obes Surg. 2007; 17:1297-305 